PUPT-1898

ITFA-99-39

SPIN-1999/29

\title{
On the Holographic Renormalization Group
}

\author{
Jan de Boer ${ }^{2,3}$, Erik Verlinde ${ }^{1}$, And Herman Verlinde ${ }^{1,4}$ \\ 1 Physics Department, Princeton University, Princeton, NJ 08544 \\ 2 Spinoza Institute, University of Utrecht, \\ Leuvenlaan 4, 3584, CE Utrecht, The Netherlands \\ ${ }^{3}$ Insituut-Lorentz for Theoretical Physics, University of Leiden, P.O.Box 9506, NL-2300 \\ RA Leiden, The Netherlands. \\ ${ }^{4}$ Institute for Theoretical Physics, University of Amsterdam, 1018 XE Amsterdam
}

\begin{abstract}
We propose a direct correspondence between the classical evolution equations of 5-d supergravity and the renormalization group (RG) equations of the dual 4-d large $N$ gauge theory. Using standard Hamilton-Jacobi theory, we derive first order flow equations for the classical supergravity action $S$, that take the usual form of the Callan-Symanzik equations, including the corrections due to the conformal anomaly. This result gives direct support for the identification of $S$ with the quantum effective action of the gauge theory. In addition we find interesting new relations between the beta-functions and the counterterms that affect the 4-d cosmological and Newton constant.
\end{abstract}




\section{Introduction}

A central element in the correspondence between classical 5-dimensional supergravity and 4-dimensional large $N$ gauge theory [1] is the interpretation of the extra 'radial' 5-th coordinate $r$ with a renormalization group parameter of the 4-d field theory [2] [3] [4]. The radial evolution of the 5-d fields indeed shares many features with an RG flow [5] [6] [7] [8] [9] [10] [11] [12], but a complete understanding of this correspondence still seems to be lacking. The purpose of this paper is to fill some of the gaps in this dictionary and to further clarify the relation between gravity and the renormalization group. Concretely, we will show that the supergravity equations of motion can be reduced to a flow equation for the classical supergravity action $S$, which furthermore, in the asymptotic limit, can be cast in the form of a standard Callan-Symanzik equation. We regard this result as additional justification for the identification of the supergravity action $S$ with the quantum effective action of the dual 4-d gauge theory. We furthermore will obtain new interesting relations between the various terms in the gravitational part of the 4-d effective action, in particular the terms that affect the Newton constant and the cosmological term. These relations suggest an intimate connection between the physics of the renormalization group and the 4-d Einstein equations. This point is worked out in more detail in a separate paper [13]. Our discussion will be quite general and will not be restricted to a particular dual pair of supergravity and (conformal) gauge theory. Although our approach is new, there is some overlap between our results and earlier papers [5] [6] [7] [8] [9] [10] [11] [12] on the holographic RG-flow.

\section{The holographic correspondence}

To set notation we begin with a quick review of the basic elements of the AdS/CFT correspondence [1] [2] [3]. The central proposal here is that there exists an exact duality between large $N$ 4-dimensional $\mathcal{N}=4$ Yang-Mills theory and type IIB string theory, or for large 't Hooft coupling, IIB supergravity living on $S_{5}$ times $A d S_{5}$. The supergravity theory contains scalars $\phi^{I}$ that represent the couplings of the 4-d gauge theory, and the propagation of these fields in the $r$-direction is believed to correspond to the 4-dimensional RG-flow. Since these fields are coupled to gravity, their stress energy will affect the shape of the background geometry, which as a result will no longer be given by the pure AdS form. To study this radial evolution it will be convenient to choose an analogue of the familiar temporal gauge, in which the 5-d metric takes the form円

$$
d s^{2}=d r^{2}+g_{\mu \nu}(x, r) d x^{\mu} d x^{\nu}
$$

The metric $g_{\mu \nu}$ and the fields $\phi^{I}$ are allowed to be dependent on all five coordinates $x^{\mu}$ and $r$.

We choose a set-up in which the 5 -d space has Euclidean signature and has a boundary at a finite value of $r$. We now define $S[\phi, g]$ as the classical action of the supergravity

\footnotetext{
${ }^{1}$ To re-introduce the lapse and shift functions one can make the substition $d r \rightarrow N d r$ and $d x^{\mu} \rightarrow$ $d x^{\mu}+N^{\mu} d r$
} 
solution with boundary values $\phi^{I}(x)$ for the fields, and $g_{\mu \nu}(x)$ for the metric. To get a unique configuration for a given choice of $\phi^{I}$ and $g_{\mu \nu}$ we further impose that the resulting five-dimensional space, even though it is geometrically different from $A d S_{5}$, still has the same topology, namely that of a five-ball $B_{5}$. By requiring that the fields $\phi^{I}$ and the metric remain regular in the interior of $B_{5}$, there is in principle one unique classical solution for given boundary values of the fields and the metric [3]. According to the prescription of [2] [3], the classical action $S[\phi, g]$ evaluated on the corresponding classical solution describes the generating function of gauge invariant operators $\mathcal{O}_{I}$ in the gauge theory

$$
\left\langle\mathcal{O}_{I_{1}}\left(x_{1}\right) \ldots \mathcal{O}_{I_{n}}\left(x_{n}\right)\right\rangle=\frac{1}{\sqrt{g}} \frac{\delta}{\delta \phi^{I_{1}}\left(x_{1}\right)} \ldots \frac{1}{\sqrt{g}} \frac{\delta}{\delta \phi^{I_{n}}\left(x_{n}\right)} S[\phi, g]
$$

When one puts the fields $\phi^{I}$ to zero after doing the variation one computes the correlators of the unperturbed $N=4$ supersymmetric Yang-Mills theory. One can also put $\phi^{I}$ equal to a finite value, which corresponds to switching on a finite perturbation of the field theory. So in principle we could consider all gauge theories that can be obtained by a general perturbation of the $N=4$ SYM theory.

Keeping the boundary at a finite radial position $r$ amounts to keeping a finite UV cutoff in the gauge theory. Before one can remove this cut-off and take the limit $r \rightarrow \infty$, one must cancel the infinities that arise in the classical action of the supergravity solution. The main source of infinities is the growing metric, or volume, at infinity, since we have $g_{\mu \nu} \sim e^{2 \lambda r} \hat{g}_{\mu \nu}$, where $\lambda$ denotes the inverse curvature radius of the asymptotic AdS-space. One of our goals will be to use the classical supergravity equations to show that, after subtracting these infinities, the resulting correlation functions satisfy the standard CallanSymanzik equations, identical in form to the one derived from the gauge theory. The idea behind this correspondence is that the infinities one finds in taking the AdS-boundary to infinity can be directly matched those of the quantum field theory calculation. In both cases the divergences can be canceled by means of a finite number of counter terms, provided one restricts to renormalizable perturbations. On the supergravity side, these renormalizable perturbations are represented by 'tachyonic' scalar fields $\phi^{I}$ with $4<\lambda^{2} m_{I}^{2}<0$. These fields decay exponentially as we approach the boundary at $r=\infty$, and therefore will not contribute to any additional infinities. In the following we will always restrict ourselves to renormalizable and marginal perturbations.

\section{Radial evolution}

The 5-d equations of motion in the gauge (11) can be written as a Hamilton system, with time replaced by the radial coordinate $r$. The hamiltonian that generates this radial flow is the analog of the familiar ADM hamiltonian

$$
H=\int d^{4} x \sqrt{g} \mathcal{H}
$$


with

$$
\mathcal{H}=\left(\pi^{\mu \nu} \pi_{\mu \nu}-\frac{1}{3} \pi_{\mu}^{\mu} \pi_{\nu}^{\nu}\right)+\frac{1}{2} \pi_{I} G^{I J}(\phi) \pi_{J}+\mathcal{L}(\phi, g)
$$

Here $\pi_{\mu \nu}$ and $\pi_{I}$ are the canonical momentum variables conjugate to $g^{\mu \nu}$ and $\phi^{I}$, and $\mathcal{L}$ denotes the local lagrangian density

$$
\mathcal{L}(\phi, g)=V(\phi)+R+\frac{1}{2} \partial^{\mu} \phi^{I} G_{I J}(\phi) \partial_{\mu} \phi^{J}
$$

with $V(\phi)$ and $G_{I J}(\phi)$ the 5-d scalar potential and metric. Here we have chosen to work in the 5-dimensional Einstein frame, so that we can use 5-d Planck units with $\kappa_{5}=1$.

The 5-dimensional supergravity equations of motion are implied by the standard Hamilton equations, supplemented with the additional constraints

$$
\nabla^{\mu} \pi_{\mu \nu}+\pi_{I} \nabla_{\nu} \phi^{I}=0
$$

as well as the Hamilton constraint

$$
\mathcal{H}=0
$$

which ensures the invariance under local shifts $r \rightarrow r+\delta r(x)$ of the 'equal time slices' $r=$ const.

\section{Hamilton-Jacobi equation}

Let us now bring the action $S[\phi, g]$ back in to our discussion. It is a standard fact, well known in classical mechanics, that the value of the canonical momentum conjugate to $\phi^{I}$ at a given 'time' $r$ is equal to the functional $\phi^{I}$ derivative of the classical action $S$, for the classical solution with boundary value $\phi^{I}(r)=\phi^{I}$. This fact also holds when the 'time' direction is replaced by a space-like coordinate. Calling this momentum variable $\pi_{I}$, we thus have

$$
\pi_{I}=\frac{1}{\sqrt{g}} \frac{\delta S}{\delta \phi^{I}} .
$$

This relation is true independent of the initial conditions or other details of the classical trajectory. Furthermore, the momentum $\pi_{I}$ is related to the flow velocity $\dot{\phi}^{I}$ by

$$
\dot{\phi}^{I}=G^{I J} \pi_{J}
$$

where $G_{I J}$ is again the 5 -d scalar metric on the space of couplings. Here the dot represents differentiation with respect to the radial coordinate $r$, even though it is actually a space-like coordinate. 
We can do the same for the metric. In classical 5-d gravity, the momentum variable $\pi_{\mu \nu}$ conjugate to $g_{\mu \nu}$ is expressed as

$$
\pi_{\mu \nu}=\frac{1}{\sqrt{g}} \frac{\delta S}{\delta g^{\mu \nu}} .
$$

and is related to the flow velocity of $g_{\mu \nu}$ viaf

$$
\dot{g}_{\mu \nu}=2 \pi_{\mu \nu}-\frac{2}{3} \pi_{\lambda}^{\lambda} g_{\mu \nu}
$$

Combining these four equations gives the first order form of the flow of the couplings $\phi^{I}$ and the metric. Given the functional form of the classical action $S[\phi, g]$ one can unambiguously compute the radial derivative of the couplings and metric in terms of their values at that point.

Let us insert the relations (10) and (11) into the constraints (6) and (7). Equation (6) gives

$$
\nabla^{\mu} \frac{\delta S}{\delta g^{\mu \nu}}+\nabla_{\nu} \phi^{I} \frac{\delta S}{\delta \phi^{I}}=0
$$

This constraint is easily satisfied: it simply means that the effective action $S$ is invariant under 4-d coordinate transformations. Equation (7) takes the form

$$
\frac{1}{\sqrt{g}}\left(\frac{1}{3}\left(g^{\mu \nu} \frac{\delta S}{\delta g^{\mu \nu}}\right)^{2}-\frac{\delta S}{\delta g^{\mu \nu}} \frac{\delta S}{\delta g_{\mu \nu}}-\frac{1}{2} G^{I J} \frac{\delta S}{\delta \phi^{I}} \frac{\delta S}{\delta \phi^{J}}\right)=\sqrt{g} \mathcal{L}(\phi, g)
$$

This Hamilton-Jacobi constraint will play a central role in the remainder. It is important to realize that it is not a constraint on the value of the variations of the action $S$ nor an equation of motion of the 4-dimensional fields: instead one must read it as a functional

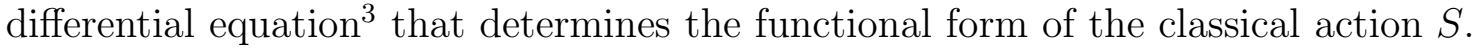

\section{Derivative expansion .}

At the scale of the cut-off $\mu_{c} \sim e^{\lambda r}$ the action $S[\phi, g]$ is non-local. But at an energy scale $\mu \ll \mu_{c}$ a part of $S$ can be represented as a local action. Hence, the action $S[\phi, g]$ can in that case be be decomposed in a local and a non-local part

$$
S[\phi, g]=S_{\mathrm{loc}}[\phi, g]+\Gamma[\phi, g]
$$

\footnotetext{
${ }^{2}$ With non-trivial lapse and shift $\dot{g}_{\mu \nu} \rightarrow N^{-1}\left(\dot{g}_{\mu \nu}-\nabla_{\mu} N_{\nu}-\nabla_{\nu} N_{\mu}\right)$.

${ }^{3}$ It is instructive to compare it to the Hamilton-Jacobi equation for a relativistic point particle: $\left(\partial_{t} S\right)^{2}-$ $\left(\partial_{x} S\right)^{2}=m^{2}$. We see that scale variation plays the role of time differentiation, which presumably is part of the reason for why the conformal factor has the 'wrong' sign in the Einstein action.
} 
with

$$
S_{\mathrm{loc}}[\phi, g]=\int \sqrt{g}\left(U(\phi)+\Phi(\phi) R+\frac{1}{2} \partial^{\mu} \phi^{I} M_{I J}(\phi) \partial_{\mu} \phi^{J}\right)
$$

where $U, \Phi$ and $M_{I J}$ are local functions of the couplings, and $\Gamma[\phi, g]$ contains all higher derivative and non-local terms. Eventually we are interested in the limit $\mu_{c} \rightarrow \infty$, or more precisely, $\epsilon=\mu / \mu_{c} \rightarrow 0$. In that limit the local potential term will contain quartic divergences, while the other local terms are quadratically divergent. In fact, even $\Gamma$ contains divergences of a logarithmic type. We further note that the local action $S_{\text {loc }}$ is similar in structure as the lagrangian term $\mathcal{L}$ in the Hamilton constraint (13). In fact, as we will see, the different terms in $S_{\text {loc }}$ have a direct relation with the corresponding terms in $\mathcal{L}$.

The idea of the following calculations will be to insert the expansion (14) into the Hamilton constraint (13), combine the contributions on the left hand side that have the same functional form as the terms on the right hand side, and require them to cancel. This procedure becomes a systematic expansion in the situation where $\mu<<\mu_{c}$, since the different local and non-local terms can then be distinguished by their scaling behavior, up to a redefinition of $\Gamma[\phi, g]$ by finite local terms. We first will focus on the potential term, which in the limit $\mu_{c} \rightarrow \infty$ would be quartically divergent. The other terms involving $\Phi$ and $M_{I J}$ will be considered in a later section.

By comparing the potential terms on both side, we derive that $U$ and the 5 -d potential term $V$ are related via

$$
V=\frac{1}{3} U^{2}-\frac{1}{2} \partial_{I} U G^{I J} \partial_{J} U
$$

Hence $V$ is expressed in terms of $U$ in the same way as if $U$ were the superpotential $W$ of the 5-d supergravity. For supersymmetric flows $U$ is indeed equal to the superpotential. However, in deriving this expression we have not made use of supersymmetry: it is simply a consequence of the standard Hamilton-Jacobi theory applied to 5-d gravity, cf. [12]. In fact, as we will show in the Appendix, different potentials $U$ could lead to the same potential $V$, and hence $U$ may in general be different from the superpotential $W$. We finally note that the relation (16) between the 4-d potential $U$ and the 5 d potential $V$ was also found to be a sufficient, and possibly necessary condition for the stability of domain wall structures in 5-d AdS gravity [1] [14.

\section{Holographic RG-flow}

Let us return to the flow equations (9) and (11). The field $\phi^{I}$ and also the metric $g_{\mu \nu}$ play a dual role in our discussion. On the one had they represent the couplings and the geometrical background of the gauge theory, while on the other hand they describe the sources for the local operators $\mathcal{O}_{I}$ and the stress-energy tensor $T_{\mu \nu}$. In standard quantum field theory one computes the beta-functions for the coupling constants in a flat background without any sources. Indeed, to compare our flow equations with the standard RG-flow we have to consider the theory at a length scale which is much longer than the cut-off scale, so 
that at the cut-off scale the fields practically are independent of the 4-d coordinates. In this limit the potential term $U$ will dominate over all other terms.

So let us write the flow equation for the scalar fields and the metric while keeping only the potential term,

$$
\dot{\phi}^{I}=G^{I J}(\phi) \partial_{J} U(\phi) \quad \dot{g}_{\mu \nu}=\frac{1}{3} U(\phi) g_{\mu \nu}
$$

We see that the metric simply rescales. Hence we can solve its flow equation by the Ansatz

$$
g_{\mu \nu}=a^{2} \widehat{g}_{\mu \nu}
$$

where $\widehat{g}_{\mu \nu}$ is independent of $r$ and the prefactor $a$ satisfies

$$
\dot{a}=\frac{1}{6} U(\phi) a .
$$

Since the parameter $a$ in fact determines the physical scale, we now replace the $r$ derivatives in the flow equations by derivatives with respect to $a$, by using the relation (19). In this way we obtain

$$
a \frac{d}{d a} \phi^{I}=\beta^{I}(\phi)
$$

where the beta-functions are defined by

$$
\beta^{I}(\phi)=\frac{6}{U(\phi)} G^{I J}(\phi) \partial_{J} U(\phi)
$$

Thus we see that the holographic RG-flow is in general derived from a potential.

\section{Relations for the local terms}

The relation (16) for the potentials $V$ and $U$ can now be re-expressed as a condition on the squares of the beta-functions:

$$
\frac{1}{24} \beta^{I} G_{I J} \beta^{J}=1-\frac{3 V}{U^{2}}
$$

Since the left hand side is positive definite, the potential $U$ obeys the inequality $U^{2} \geq 3 V$, where the equal sign only holds at fixed points of the RG-flow. The relations between the other local terms in the action are also conveniently expressed in terms of the beta-functions. Comparing the curvature and kinetic terms on the right- and left-hand side gives

$$
\beta^{K} \partial_{K} \Phi=2 \Phi+\frac{6}{U}
$$


and

$$
\beta^{K} \partial_{K} M_{I J}-\beta^{K} \partial_{I} M_{K J}-\beta^{K} \partial_{J} M_{I K}=2 M_{I J}+\frac{6}{U} G_{I J}
$$

In addition, there is also a term on the left-hand side of (13) proportional to $\nabla^{2} \phi$ that is not present on the right-hand side. Requiring the coefficient in front of it cancels gives another interesting expression for the beta-function in terms of purely 4-d quantities

$$
\beta^{I}=M^{I J} \partial_{J} \Phi
$$

In most studies of the AdS/CFT correspondence, the local terms of the boundary effective action are considered to be non-universal, since they diverge as we take the limit $r \rightarrow \infty$. Here we see, however, that they contain very important information about the flow of the couplings constants, and even conspire in an interesting way to make up the lagrangian term $\mathcal{L}$ of the 5 -d gravity theory.

\section{Callan-Symanzik equation}

As was mentioned before, the action $S$ represents the generating function of correlation functions of local operators $\mathcal{O}_{I}$ in the gauge theory. In fact, the information about $n$-point functions of operators $\mathcal{O}_{I}$ at different points is all contained in the non-local part of the action $\Gamma$. We already established various relations between the local terms. Following the same strategy we will now derive a flow equation for the correlation functions that exactly take the form of Callan-Symanzik equations.

The computation proceeds as follows: we again insert the decomposition (14) into the constraint (13), and drop all local terms with two or fewer derivatives. At the order we are interested in, there are cross terms that involve (functional derivatives of) the potential $U$ and the non-local effective action $\Gamma$. At that same order one also finds curvature squared terms and products of the curvature with space-time derivatives of the scalar fields. Here we will not write these terms explicitly. The relation we then find is

$$
\frac{1}{\sqrt{g}}\left(g^{\mu \nu} \frac{\delta}{\delta g^{\mu \nu}}-\beta^{I}(\phi) \frac{\delta}{\delta \phi^{I}}\right) \Gamma[\phi, g]=\text { 4-derivative terms }
$$

In order to derive the Callan-Symanzik equations for expectation values of local operators we vary this relation with respect to fields $\phi^{I}$. After doing the variations, the fields are put to their constant average value given by the couplings of the gauge theory. We further take the metric to be of the form $g_{\mu \nu}=a^{2} \eta_{\mu \nu}$, where $a$ is $x^{\mu}$-independent. The 4-derivative terms will drop out after this step, and play no role as long as one considers operators $\mathcal{O}_{I}$ at different points in space. Finally, we integrate the resulting expression over all of space and replace the functional derivatives by ordinary derivatives by using the definitions

$$
\int g^{\mu \nu} \frac{\delta}{\delta g^{\mu \nu}}=a \frac{\partial}{\partial a}, \quad \quad \int \frac{\delta}{\delta \phi^{I}}=\frac{\partial}{\partial \phi^{I}}
$$


In this way one derives after some straightforward algebra from (26) the standard form of the Callan-Symanzik equations

$$
\left(a \frac{\partial}{\partial a}-\beta^{I} \partial_{I}\right)\left\langle\mathcal{O}_{I_{1}}\left(x_{1}\right) \ldots \mathcal{O}_{I_{n}}\left(x_{n}\right)\right\rangle-\sum_{i=1}^{n} \gamma_{I_{i}}^{J_{i}}\left\langle\mathcal{O}_{I_{1}}\left(x_{1}\right) . . \mathcal{O}_{J_{i}}\left(x_{i}\right) . . \mathcal{O}_{I_{n}}\left(x_{n}\right)\right\rangle=0
$$

wheref

$$
\gamma_{I}^{J}=\nabla_{I} \beta^{J}
$$

represent the anomalous scaling dimensions of the operators $\mathcal{O}_{I}$. We should note, however, that this equation is derived still with a finite cut-off. We will describe in a moment how one can remove the cut-off and obtain the C-S equation for the full renormalized $n$-point functions.

\section{The conformal anomaly}

In order to recover the conformal anomaly, let us relax the condition that the metric is flat, and use the constraint (13) to compute an expression for the trace of the finite part of the expectation value of the stress tensor:

$$
\left\langle T_{\mu \nu}\right\rangle=\frac{1}{\sqrt{-g}} \frac{\delta \Gamma}{\delta g^{\mu \nu}}
$$

The calculation is again similar as outlined above. We find that the trace anomaly takes form

$$
\langle T\rangle=\beta^{I} \partial_{I} \Gamma+c R^{\mu \nu} R_{\mu \nu}-d R^{2}
$$

where $T=T_{\mu}^{\mu}$, and the curvature squared terms simply arise due to the square of the Einstein term in the action $S_{\text {loc }}$. We recognize this equation as a standard type expression as dictated by the broken scale invariance of the effective action $\Gamma$ and the trace anomaly relation. If The coefficients $c$ and $d$ are given in terms of $\Omega$ and $\Phi$ by

$$
c=\frac{6 \Phi^{2}}{U}, \quad d=\frac{2}{U}\left(\Phi^{2}-\frac{3}{2} \partial_{I} \Phi G^{I J} \partial_{J} \Phi\right)
$$

We can perform a quantitative check on these coefficients, by going to the fixed point situation, where all $\partial_{I}$ derivative vanish. A simple calculation shows we can then express $c$ and

\footnotetext{
${ }^{4}$ By an appropriate choice of contact term, the ordinary $\phi^{I}$ derivative is turned in a covariant derivative $\nabla_{I}$ that is defined in terms of the metric $G_{I J}$. This ensure that the whole formalism stays covariant under field redefinitions.

${ }^{5}$ For a general 4-d conformal field theory one would also have a term given by the square of the Weyl tensor. In order to reproduce such a term one would have to add it by hand to the Hamilton constraint, as a higher order correction to the 5-d Einstein action.
} 
$d$ in terms of the 5 -d potential term $V$ as $c=3 d=(V / 3)^{-3 / 2}=\lambda^{3} / 8$, which reproduces the expression of the holographic Weyl anomaly obtained in [17]. The function $c$ is the analogue of the central charge of the 4-d QFT, and has been proposed as a candidate $C$-function in [8] and [7].

\section{Removing the UV cut-off}

So far we studied the RG-flow of the couplings, action, and correlation functions as a function of the UV cut-off. To make contact with more standard type RG-equations, we have to follow the usual renormalization procedure of cancelling divergences, introducing renormalized couplings, and then sending the cut-off to infinity. Here we will outline how this procedure works in the present context. In particular we will consider how (28) can be rewritten as the standard Callan-Symanzik equation for the renormalized $n$-point function.

Removal of the cut-off amounts to taking the limit $r \rightarrow \infty$. A practical way to describe this limit is to write the metric and the couplings as

$$
g_{\mu \nu}=\epsilon^{-2} g_{\mu \nu}^{\mathrm{R}}
$$

and

$$
\phi^{I}=\phi^{I}\left(\phi_{\mathrm{R}}, \epsilon\right)
$$

where $g_{\mu \nu}^{R}$ and $\phi_{\mathrm{R}}^{I}$ are the renormalized metric and couplings which are kept fixed as we send $\epsilon \rightarrow 0$. The relation between the bare couplings $\phi^{I}$ and the renormalized couplings $\phi_{\mathrm{R}}^{I}$ is obtained by integrating the RG-flow

$$
\epsilon \frac{\partial \phi^{I}}{\partial \epsilon}=\beta^{I}(\phi) \quad \phi^{I}=\phi_{\mathrm{R}}^{I} \quad \text { at } \epsilon=1
$$

Form the supergravity perspective, this procedure for introducing the renormalized couplings means the following. Consider the unique classical supergravity trajectory with asymptotic boundary conditions specified by the 'bare' fields $\left(\phi^{I}, g\right)$. The renormalized fields $\left(\phi_{\mathrm{R}}^{I}, g_{\mathrm{R}}\right)$ then represent the values of the scalar field on this trajectory at some finite value of the scale factor, corresponding to some fixed RG scale.

As we consider only relevant perturbations, the couplings $\phi^{I}$ will actually go to zero as $\epsilon^{\lambda_{I} / \lambda}$, as we take the limit $\epsilon \rightarrow 0$ while keeping $\phi_{\mathrm{R}}$ fixed. The metric $g_{\mu \nu}$ on the other hand diverges. Hence we still find, upon inserting the expressions (33) and (34) into the action $S$, that the various terms contained in $S_{\text {loc }}$ diverge in the limit $\epsilon \rightarrow 0$. For example, the potential term $U$ will in general be quartically divergent, while $\Phi$ and $M_{I J}$ contain at most quadratic divergences. Even the term $\Gamma$ has a potential logarithmic divergence that has to be removed in the renormalization procedure. This can be done by adding appropriate counterterms. The renormalized effective action $\Gamma_{R}$ is defined by

$$
\Gamma_{\mathrm{R}}\left[\phi_{\mathrm{R}}, g_{\mathrm{R}}\right]=\lim _{\epsilon \rightarrow 0} \Gamma_{\text {finite }}\left[\phi\left(\phi_{\mathrm{R}}, \epsilon\right), \epsilon^{-2} g_{\mathrm{R}}\right]
$$


where $\Gamma_{\text {finite }}$ is obtained from $\Gamma$ by subtracting its divergent part.

We now would like to show that the action $\Gamma_{\mathrm{R}}$ again satisfies a similar Callan-Symanzik equation as before, but now expressed in terms of the renormalized couplings and metric

$$
\frac{1}{\sqrt{g}}\left(g_{\mathrm{R}}^{\mu \nu} \frac{\delta}{\delta g_{\mathrm{R}}^{\mu \nu}}-\beta_{\mathrm{R}}^{I}\left(\phi_{\mathrm{R}}^{I}\right) \frac{\delta}{\delta \phi_{\mathrm{R}}^{I}}\right) \Gamma_{\mathrm{R}}\left[\phi_{\mathrm{R}}, g_{\mathrm{R}}\right]=\text { local terms }
$$

The derivation of this relation is basically a change of variables from $\phi^{I}$ to $\phi_{\mathrm{R}}^{I}$. The betafunctions behave as vector fields on the space of couplings, and simply transform accordingly. This can be seen as follows. Since the relation between $\phi^{I}$ and $\phi_{\mathrm{R}}^{I}$ can also be obtained by flowing down, the beta-function $\beta_{\mathrm{R}}^{I}$ should be such that the variation of $\phi^{I}$ due to an infinitesimal shift in the cut-off $\epsilon$ while keeping $\phi_{\mathrm{R}}^{I}$ constant, can be exactly compensated by an infinitesimal RG transformation of $\phi_{\mathrm{R}}^{I}$ :

$$
\epsilon \frac{\partial \phi^{I}}{\partial \epsilon}=\beta_{\mathrm{R}}^{J} \frac{\partial \phi^{I}}{\partial \phi_{\mathrm{R}}^{J}}
$$

By comparing this relation with (35) one indeed finds the usual transformation properties of a vector field. This implies that we can directly make the substitution

$$
\beta^{I} \frac{\delta}{\delta \phi^{I}}=\beta_{\mathrm{R}}^{I} \frac{\delta}{\delta \phi_{\mathrm{R}}^{I}}
$$

Furthermore, since the metric $g_{\mu \nu}^{\mathrm{R}}$ and $g_{\mu \nu}$ are related by a constant rescaling, one can simply relate their functional derivatives as well. Finally, the operators $\mathcal{O}_{I}$ also have to be renormalized, in order for their correlators to stay finite. We have

$$
\mathcal{O}_{I}^{\mathrm{R}}=\mathcal{O}_{J} \frac{\partial \phi^{J}}{\partial \phi_{\mathrm{R}}^{I}}
$$

With these substitutions it is straightforward to recover the Callan-Symanzik equations for all renormalized $n$-point functions.

$$
\left(a \frac{\partial}{\partial a}-\beta_{\mathrm{R}}^{I} \partial_{I}\right)\left\langle\mathcal{O}_{I_{1}}^{\mathrm{R}}\left(x_{1}\right) \ldots \mathcal{O}_{I_{n}}^{\mathrm{R}}\left(x_{n}\right)\right\rangle-\sum_{i=1}^{n}\left(\gamma_{\mathrm{R}}\right)_{I_{i}}^{J_{i}}\left\langle\mathcal{O}_{I_{1}}^{\mathrm{R}}\left(x_{1}\right) . . \mathcal{O}_{J_{i}}^{\mathrm{R}}\left(x_{i}\right) . . \mathcal{O}_{I_{n}}^{\mathrm{R}}\left(x_{n}\right)\right\rangle=0
$$

The above procedure for defining the renormalized beta-functions is quite standard. As presented, however, it may not be entirely obvious that the beta-functions at a given scale $\mu$ can in fact be extracted from just the knowledge of the UV physics at smaller scales. To see how this arises from the supergravity perspective, recall that $\phi_{\mathrm{R}}^{I}$ represents the value of the scalar field on a specified classical supergravity trajectory. Once we know the values of 
the bare fields as well as the initial velocities at the cut-off scale, we can integrate inwards and obtain the renormalized beta-functions $\beta_{\mathrm{R}}^{I}\left(\phi_{\mathrm{R}}\right)$ as the radial velocities (as measured relative to the overall scale of the metric) of the fields at this point. This procedure requires knowledge of the supergravity solution in the outside region only.

\section{Discussion and outlook}

In this paper we have shown that the Hamilton-Jacobi equations for 5d Einstein gravity can be written in the form of first order RG-flow equations. These results can in principle be applied to every $4 \mathrm{~d}$ gauge theory that can be represented as a relevant or marginal perturbation of a large $N$ superconformal field theory, or any of its variations for which an AdS/CFT correspondence has been established. Also, generalizations to other dimensions are straightforward. In deriving the Callan-Symanzik equations we made use of the fact that asymptotically the metric and scalar fields behave as in AdS-space, but the HamiltonJacobi equations themselves are valid in much more general situations. For example, it is conceivable that the RG-flow of practically any large $N$ gauge theory is, in leading order in $\frac{1}{N}$, described by classical supergravity equations of this type.

The relation between the $5 \mathrm{~d}$ Einstein equation, the RG-flow and Callan-Symanzik equation, as presented in the main text, was derived in the special gauge with fixed 'lapse' and 'shift' functions. Reintroducing the lapse and shifts would presumably lead to RG-equations that are invariant under local redefinitions of the scale as well as scale-dependent coordinate transformations. Together these invariances reflect the underlying 5-d general coordinate invariance of the dual supergravity theory.

It would be interesting to identify the modifications of our equations that are required to incorporate $1 / N$ corrections. The Hamilton-Jacobi equations can be considered as the classical limit of the quantum Wheeler-DeWitt equation, which (when the WDW wave-function is written as $e^{\frac{i}{\hbar} S}$ ) contains an additional term proportional to a second order variation of the action $S$. The resulting equation has a striking similarity with Polchinski's version of the exact renormalization group [15]. We believe that this is not a coincidence, since it should describe the RG-flow of the regularized (through string theory) quantum supergravity theory.

The internal 5-manifold that features in the usual AdS/CFT framework has not played an explicit role in our discussion, but its geometry is implicitly present in the form of the scalar fields $\phi^{I}$ and the metric $G_{I J}$. So the RG-flow equations in principle also describe the behavior of this manifold as one flows towards the infra-red.

Finally, it is an interesting question whether the holographic description of the RGflow that we presented in this paper also applies to 4-d gauge theories that are coupled to gravity. It has been argued in [16] that one needs to replace the asymptotic AdS geometry be a compact internal manifold, as would naturally arise in warped compactifications that give rise to 4 -d gauge theories with gravity. This leads to an interesting interplay between the 4-d Einstein equations and the RG-flow equations, that in particular seems to shed new light on the role of the cosmological constant. This direction is further investigated in [13] . 


\section{ACKNOWLEDGEMENTS}

This work is supported by NSF-grant 98-02484, a Pionier fellowship of NWO, the Packard foundation and the stichting FOM. We would like to thank V. Balasubramanian, R. Dijkgraaf, S. Kachru, I. Klebanov, G. Lifschytz, V. Periwal, A. Polyakov, L. Randall, C. Schmidhuber, E. Silverstein, K. Skenderis for helpful discussions.

\section{Appendix: Comment on the Hamilton-Jacobi relation for the potential.}

In this Appendix we study the relation between the potentials $U$ and $V$ that follows from the Hamilton-Jacobi equation in more detail. For simplicity we go to a basis in which the metric $G_{I J}$ is given by $\delta_{I J}$, so that we can write the relation as

$$
\frac{1}{3} U^{2}-\frac{1}{2}\left(\partial_{I} U\right)^{2}=V
$$

One might wonder whether any potential $V$ can be written in this form. Our derivation of this relation did not assume any special properties of $V$, except the existence of a classical solution that can be extended from the boundary to the interior. This of course puts restrictions on the potential $V$, and apparently forces it to be of the supersymmetric form. We will show in this appendix that the $4 \mathrm{~d}$ potential $U$ is for a large part, but not entirely, determined by the 5 d potential $V$.

Let us expand the 5 -d potential in powers of $\phi^{I}$ as

$$
V=12 \lambda^{2}-\frac{1}{2} m_{I}^{2} \phi^{I} \phi^{I}+g_{I J K} \phi^{I} \phi^{J} \phi^{K} \ldots
$$

where we used the freedom to shift the fields to remove a possible linear term in $\phi^{I}$. To obtain a solution to (A.1) for the 4-d potential, we first try a similar expansion.

$$
U=6 \lambda+\frac{1}{2} \lambda_{I} \phi^{I} \phi^{I}+\lambda_{I J K} \phi^{I} \phi^{J} \phi^{K}
$$

Here we already fixed the constant term so that it matches with that of $V$. The beta-functions derived from this potential are

$$
\beta^{I}=\left(4-\Delta_{I}\right) \phi^{I}+c^{I}{ }_{J K} \phi^{J} \phi^{K}
$$

where

$$
\Delta_{I}=4-\frac{\lambda_{I}}{\lambda} \quad c_{I J K}=\frac{3}{\lambda} \lambda_{I J K}
$$


are the scaling dimensions and operator product coefficients of the operators $O_{I}$ corresponding to the couplings $\phi^{I}$. Inserting both expansions (A.2) and (A.3) in to (A.1) then gives the relation

$$
\lambda_{I}^{2}+4 \lambda \lambda_{I}=m_{I}^{2}
$$

which upon inserting the relation (A.5) is recognized as standard relation between the scale dimensions $\Delta_{I}$ of the 4-d couplings and the corresponding masses $m_{I}$ of the 5-d fields [2] [3]. Notice that this relation implies that the 5-d potential must in fact satisfy the unitarity bound $m_{I}^{2} \geq-4 \lambda^{2}$. If this inequality is violated, there are no bounded solutions that extend all the way to the asymptotic boundary.

By looking at the next order in the $\phi^{I}$ expansion we obtain the relation

$$
\left(\lambda_{I}+\lambda_{J}+\lambda_{K}-4 \lambda\right) \lambda_{I J K}=g_{I J K}
$$

which via (A.5) expresses the operator product coefficients $c_{I J K}$ in terms of the cubic term in $V$. Note that this expression degenerates in the case that $\Delta_{I}+\Delta_{J}+\Delta_{k}=8$. The interpretation of this will become clear when we look at deformations of $U$ that preserve the relation (A.1) with $V$.

An infinitesimal variation $\delta U$ preserves (A.1) if it satisfies the linear relation

$$
4 U \delta U-6 \partial_{I} U \partial_{I}(\delta U)=0
$$

or

$$
\left(4-\beta^{I} \partial_{I}\right) \delta U=0
$$

These equations tell us that those terms in $U$ that have total dimension 4 are not determined by the Hamilton-Jacobi constraint. It is interesting to note that these are precisely those terms that remain finite in the continuum limit. Therefore, it appears that the HamiltonJacobi relation only constrains the divergent terms of the potential $U$, but not the finite part.

\section{References}

[1] J. Maldacena, The large $N$ limit of supercomformal field theories and supergravity, Adv. Theor. Math. Phys. 2 (1998) 231, hep-th/9711200.

[2] S. Gubser, I. Klebanov and A. Polyakov, Gauge Theory Correlators from Non-Critical String Theory, Phys.Lett.B428 (1998) 105, hep-th/9802109;

[3] E. Witten, Anti-de Sitter Space and Holography, Adv.Theor.Math.Phys.2 (1998) 253, hep-th/9802150; 
[4] L. Susskind and E. Witten, The Holographic Bound in Anti-de Sitter Space, hepth/9805114

[5] E.T. Akhmedov, A Remark on the AdS/CFT Correspondence and the Renormalization Group Flow, Phys. Lett. B442 (1998) 152, hep-th/9806217.

[6] E. Alvarez and C. Gómez Geometric Holography, the Renormalization Group and the c-Theorem, Nucl. Phys. B541 (1999) 441, hep-th/9807226.

[7] D. Z. Freedman, S. S. Gubser, K. Pilch, N. P. Warner, Renormalization Group Flows from Holography-Supersymmetry and a c-Theorem, hep-th/9906194.

[8] L. Girardello, M. Petrini, M. Porrati, A. Zaffaroni, The Supergravity Dual of $N=1$ Super Yang-Mills Theory, hep-th/9909047.

[9] L. Girardello, M. Petrini, M. Porrati, A. Zaffaroni, Novel Local CFT and Exact Results on Perturbations of $N=4$ Super Yang Mills from AdS Dynamics, hep-th/9810126;

M. Porrati, A. Starinets, RG Fixed Points in Supergravity Duals of 4-d Field Theory and Asymptotically AdS Spaces, Phys.Lett. B454 (1999) 77, hep-th/9903241.

[10] V. Balasubramanian, P. Kraus, Space-time and the Holographic Renormalization Group, Phys.Rev.Lett 83 (1999) 3605, hep-th/9903190,

[11] K. Skenderis, P.K. Townsend, Gravitational Stability and Renormalization-Group Flow, hep-th/9909070.

[12] O. DeWolfe, D.Z. Freedman, S.S. Gubser, A. Karch, Modelling the fifth dimension with scalars and gravity, hep-th/9909134.

[13] E. Verlinde and H. Verlinde, RG Flow, gravity, and the cosmological constant, to appear.

[14] K. Behrndt, M. Cvetic, Supersymmetric Domain-Wall World from D=5 Simple Gauged Supergravity, hep-th/9909058;

U. Ellwanger, Constraints on a Brane-World from the Vanishing of the Cosmological Constant, hep-th/9909103;

[15] J. Polchinski, Renormalization and Effective Lagrangians, Nucl. Phys, B231 (1984) 269.

[16] H. Verlinde, Holography and compactification, hep-th/9906182; L. Randall and R. Sundrum, A Large Mass Hierarchy from a Small Extra Dimension, hep-ph/9905221; An Alternative to Compactification, hep-th/9906064.

[17] M. Henningson and K. Skenderis, The Holographic Weyl Anomaly, JHEP 9807:023 (1998), hep-th/9806087. 